\title{
The Role of Physical Agents in Treatment of Osteoporosis
}

\author{
Erieta Nikolikj-Dimitrova* \\ Institute of Physical Medicine and Rehabilitation, Faculty of Medicine, Ss Cyril and Methodius University, Skopje, Republic of \\ Macedonia
}

\begin{abstract}
Citation: Nikolikj-Dimitrova E. The Role of Physical Agents in Treatment of Osteoporosis. Maced J Med Sci. 2013 June 15; 6(2):189-193. http://dx.doi.org/10.3889/MJMS.1857-5773.2013.0286. Key words: osteoporosis; physical agents; pulsed electromagnetic field; ultraviolet rays.

"Correspondence: Erieta Nikolikj-Dimitrova $\mathrm{MD}, \mathrm{PhD}$. Institute of Physical Medicine and Rehabilitation, Elisie Popovski 28, 1000 Skopje, Republic of Macedonia. Phone: 077646 600. EMail: erietand@yahoo.com

Received: 14-Sep-2012; Accepted: 05-Mar2013; Online first: 13-Mar-2013

Copyright: (c) 2013 Nikolikj-Dimitrova E. This is an open-access article distributed under the terms of the Creative Commons Attribution License, which permits unrestricted use distribution, and reproduction in any medium, provided the original author and source are provided the original author and source are
credited.

Competing Interests: The authors have declared that no competing interests exist.
\end{abstract}

\begin{abstract}
Osteoporosis is a chronic, progressive disease, which represents a multifactorial condition. Treatment of patients with osteoporosis has several aspects that require a team approach. It consists of application of appropriate drug therapy, education, diet, exercises and physical therapy.

Aim of the study is to present physical agents applicable in the prevention and treatment of people suffering from osteoporosis.

Physical agencies that can be applied in prophylaxis of osteoporosis are heliotherapy and ultraviolet radiation. For osteoporosis treatment ultraviolet rays, a low-frequency impulse magnetic field, middle-frequency interferential currents could be applied. For reducing pain transcutaneous electrical nerve stimulation, ultrasound, horizontal therapy, diadynamic currents can be used.

Conclusion: To complement pharmacological therapy and exercises applied in the complex treatment of patients with osteoporosis, specific physical agents should also take their place and contribute in reducing subjective symptoms, improving clinical findings and the quality of life. However, further clinical studies are required to achieve a better record on their application.
\end{abstract}

\section{Introduction}

Osteoporosis is a systemic skeletal disease characterized by low bone mass and microarchitectural deterioration of bone tissue, with a consequent increase in bone fragility and susceptibility to fractures [1].

It is a chronic, progressive disease, which represents a multifactorial condition. The disease has usually no specific symptoms to be recognizable as osteoporosis. The most common clinical manifestations are in broken bones leading to emergence of pain, disability, restrictions in activities of daily living, reduced quality of life, invalidity and increased need for health care [1].

Pain is usually associated with fracture, which is often a low energy fracture occurring in the vertebral bodies, distal part of radius and at the femur neck. Patients with gradual occurrence of compressive fractures in the anterior part of the vertebral bodies have increased thoracic kyphosis that can be followed by chronic back pain. Kyphosis causes bad posture of spine and changes in the center of gravity of the body that increases the risk of falling.

Chronic back pain that occurs after previous vertebral compressive fractures is considered to be a consequence of skeleton deformity, joint incongruence and tension in the muscle-tendon structures [2]. Back pain adversely affects the quality of life for these patients [3].

The aim of the study is to present physical agents applicable in the prevention and treatment of people suffering from osteoporosis.

\section{Non-pharmacological treatment of osteoporosis}

Treatment of patients with osteoporosis has several aspects that require a team approach. Endocrinologist, orthopedist, physiatrist, physiothera- 
pist and other experts have a significant place in the diagnostic and treatment procedures of osteoporosis.

Due to high costs involved in the treatment of patients diagnosed with osteoporosis and its complications, which are high both for the family budget and for society, great importance is attached to prevention, early detection and early initiation of treatment, and to changes in lifestyle in order to reduce the risk of fractures.

Bone loss prevention should be a major objective in primary and secondary prevention. Specific measures should be taken as early as in puberty, adolescence and in young adults to promote bone formation and to achieve maximum bone mass through combination of exercises and appropriate diet with Calcium and Vitamin D [4].

Prevention in adults should also be implemented through education, detection of risk factors, regular controls, advices on Calcium and Vitamin D rich diet, regular exercises and physical activities that increase mineral bone density, including exercises for balance, elasticity, flexibility and coordination.

Treatment of patients with osteoporosis consists of application of appropriate conventional pharmacological therapy, diet, and education. To complement it, non-pharmacological treatment options have been developed in the last decades like exercises and physical therapy.

There are a number of published studies pointing out the effects of application of pharmacological therapy.

Several published systematic reviews demonstrate that physical exercise programs including impact exercises, specific strength training, balance and coordination training may maintain or increase spine and hip bone mineral density as well as decrease the frequency of falls among osteoporotic and osteopenic patients $[5,6,7]$.

Aim of the physical therapy in patients with osteoporosis is to maintain bone mass, stimulate the process of calcium deposits in bones, prophylaxis and treatment of spine deformities, and reduction of pain [8]. Physical therapy is believed to slow down the progression of process, reduce symptoms, especially pain, and improve mobility and quality of life [9].

Physical agencies that can be applied in prophylaxis of osteoporosis are the following: heliotherapy (adequate exposure to sunlight) that stimulates $\mathrm{D}$ Vitamin creation, ultraviolet radiation (UVR) in below minimal erythema dose in autumnwinter periods [8, 10], and climate therapy [8].

To stimulate osteogenesis, below minimal erythema dose of ultraviolet rays and dozing heliotherapy are applied. In order to influence microcirculation in patients with osteoporosis, lowfrequency pulsed electromagnetic field, low-frequency currents with vasodilatation and trophic parameters (diadynamic currents - modulations DF, CP, LP), middle-frequency interferential currents with constant frequency of $100 \mathrm{~Hz}$ or rhythmic frequency 1-100 Hz with trophic action could be applied (Figure 1) [8].

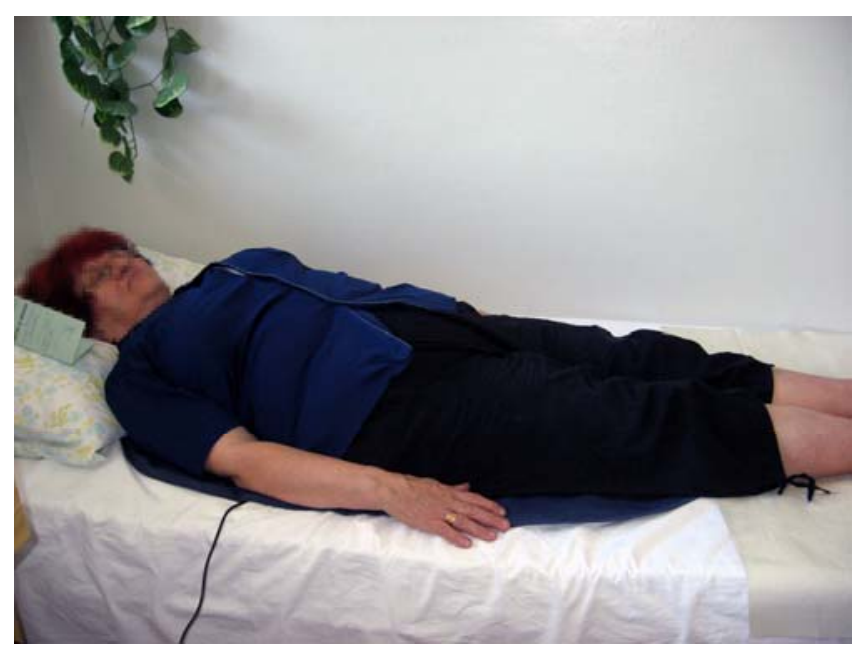

Figure 1: Application of low-frequency pulsed electromagnetic field in thoracic-lumbar region.

Also, vibratory platforms $[11,12]$, low intensity electrical stimulation, laser therapy and ultrasound show positive effects on osteoporotic tissue [11].

Electromagnetic fields influence every cell in the body in the region where applied. Magnetic fields change permeability of the cell membrane and affects ion balance, change orientation of the dipole molecules and distribution of ions across a cell membrane, affect the diffusion process and active transport through cell membrane, have effects on the interaction of receptors of cell membrane, improve oxygenation and metabolism in tissues, activate oseoblasts, hondroblasts and fibroblasts, activate enzyme systems, have analgesic effects, etc. [8, 10].

In the case of acute pain physiotherapy fulfils the function of immediate therapy (rest and "mild" cold applications) [13].

Application of TENS is recommended in patients with pain, usually the conventional one [13], interferential currents constant $100 \mathrm{~Hz}$, diadynamic currents and Trabert's currents, ultrasound may also be applied, with a constant or impulse regime, a labile method in a doze of 0,3 to $0,6 \mathrm{~W} / \mathrm{cm}^{2}$ (Figure 2) [8, 10].

Other authors recommend application of thermic (hydrothermic, high frequency thermic, light thermic) and mechanical (massage) stimuli, which can be applied regionally, locally or holistically [13].

Physiotherapy in osteoporosis essentially takes the form of stimulatory therapy tailored to the findings and the pathomechanism. The choice of therapy and its dosage depend on the desired result (prevention, cure, and rehabilitation) [13]. 


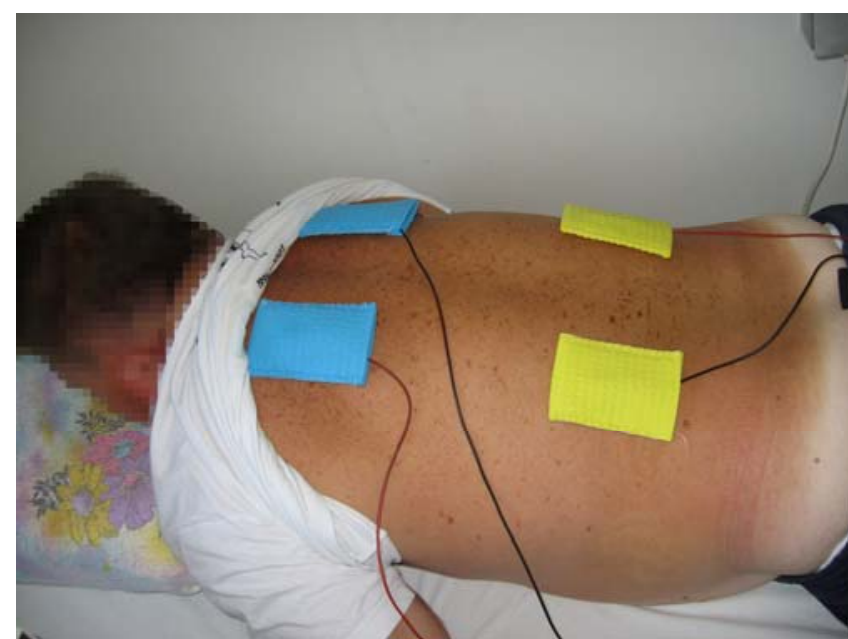

Figure 2: Application of interferential currents in thoracic region.

Physical therapy is by no means used in the rehabilitation of patients after conservatively or operatively treated fractures due to osteoporosis.

\section{Experimental studies' results}

There are few published results on experimental, and even fewer on clinical studies analyzing the effects of application of physical agents in patients with osteoporosis.

Results of experimental studies in animals indicated that the use of pulsed electromagnetic fields (PEMF) 1 hour per day influenced the activities of osteoblasts, whereby maximal osteogenetic effects were achieved with a doze of 0.01 and $0.04 \mathrm{~T}$ per second. Maximal osteogenetic response was confirmed by reduction of the level of intracortical remodeling, inhibition of endosteal resorption and by stimulation of periosteal and endosteal formation of new bone [14].

An experimental study in 18 ovariectomized rats investigated preventive effects of low-frequency $(50 \mathrm{~Hz})$ electromagnetic fields (EMF) with low intensity $(1 \mathrm{mT})$ and longer use in a period of 6 weeks. Mineralization and morphology of tibia was tested. In the group exposed to EMF, thickening of the tibial cortex considerably increased levels of $\mathrm{Na}$ and $\mathrm{K}$ in tibia were noticed, as well as a considerably increased level of alkaline phosphatase. The results supported the belief that the EMF application can be an efficient method in treatment of osteoporosis and other conditions related in loss of bone tissue [15].

Another study investigated the effect of lowfrequency electromagnetic fields with low strength in a group of 35 three-months-old rats, which were bilaterally ovariectomized. The histomorphologic results showed that the stimulation with PEMF extended and restored the bone mass of metaphyseal trabacular bone in the proximal part of tibia (increased percentage of hard tissue, percentage of bone volume and number of trabecules) and the architecture (increased trabecular perimeter, thickening of trabacules and reduced separation of trabacules) in a group exposed to PEMF. The trabacular bone mass in animals stimulated with PEMF for a period of 30 days was recovered to the level of age of the intact rats. The PEMF stimulation affected the increase of prostaglandin $E(2)$ serum concentration and the achievement of its level same as with the intact rats. The authors concluded that PEMF might be useful in the prevention of ovariectomy-induced osteoporosis and that perhaps the prostaglandin $E(2)$ level had to do with such preventive effects [16].

An experimental study using in vitro culture model of osteoblasts investigated the effects of lowfrequency $(7.5 \mathrm{~Hz})$ PEMF on stimulation of osteoblast population, citokins [prostaglandin $E(2)$ ], the transforming growth factor -beta1 and activity of alkaline phosphatase to find an optimal intensity of PEMF that affects the growth of osteoblasts. The results showed that PEMF could stimulate the growth of osteoblasts, the release of the transforming growth factor-beta1 and thus increase the activity of alkaline phosphatase. The synthesis of prostaglandine $E(2)$ release in culture medium was reduced by the increase in the number of cells. Higher PEMF intensity does not necessarily mean an increased growth of osteobasts, whereby in this case the most efficient intensity was about $2 \mathrm{mV} / \mathrm{cm}$. The authors believed that lower dozes of PEMF should be determined, and the study results have slightly shed light on the mechanism of PEMF stimulation in the therapy of fractures that have not healed and in the prevention of osteoporosis [17].

A recent experimental study examined the effects of PEMF on bone mineral density in rats. The results showed that bone mineral density and concentrations of the transforming factor of growth beta 1 considerably increased after 8 weeks in the group receiving PEMF treatment. The level of interleukin- 6 was considerably reduced in the PEMF group in comparison with the group having an inactive osteoporosis. The results obtained demonstrated that the PEMF stimulation could effectively suppress the loss of bone mass. The authors concluded that PEMF could influence the process of bone remodeling by promoting secretion of the transforming growth factorbeta1 and inhibition of expression of interleukin-6 [18].

Recently, low level laser therapy (LLLT) shows stimulatory effects on osteoblast-like cells, increasing its viability [19], DNA and RNA synthesis, bone nodule formation [20].

\section{Clinical studies' results}

A clinical study examined the effects of intermittent compressive therapy that increase blood flow in the leg over bone mineral density at femur neck in menopausal women. The study included 37 women with low bone mineral density (BMD, t score<- 
1) at femur neck or at lumbar spine, of which 24 completed the tests. These women were treated with intermittent pneumatic compression 2 hours a day for a period of 6 months, alternately to one and to the other leg. Investigations lasted for 1 year. The women received $1 \mathrm{gr}$. Calcium and $800 \mathrm{IE}$ Vitamin D daily. After 12 months, the BMD of lumbar spine was reduced by $0.5 \%$, at the right and left femur neck slightly increased by $3 \%$ and $2 \%$, and there was no change at the distal part of tibia or heals. The analysis of interaction between exercises and changes in BMD showed statistically increase of BMD at femur neck after 6 months of intermittent compression on the right leg, however, only in women with minimal exercise. The authors concluded that the preliminary results could suggest that the intermittent pneumatic leg compression could have a role in preventing osteoporosis, particularly in women with a sedentary lifestyle [21].

Huan $L$ et. al. in a literature review presented their understanding of the effects of low-frequency PEMF on chronic bone pain, bone mineral density (BMD), bone strength and biochemical markers of bone metabolism in patients with osteoporosis. For that purpose, PubMed databases were searched for appropriate studies published in English in China Knowledge Infrastructure (CNKI) for studies published in the Chinese language in the period from January 1996 to December 2007. Studies on the effects of PEMF in primary and secondary osteoporosis where searched in, either observational or control studies. A total number of 111studies was found, of which 101 published in Chinese, and 10 in English, out of which only 34 studies met the analysis criteria. The conclusions of the authors were that the lowfrequency PEMF reduced pain in primary osteoporosis quickly and efficiently, stimulated bone formation and increased bone mineral density in secondary osteoporosis. However, the effects of lowfrequency PEMF on bone mineral density in primary osteoporosis and on bone resorption were controversial [22].

As some of the physical agents have good analgesic effects and at the same time can have a positive trophic effect, they could be applied to reduce back pain that usually is chronic and thus improve the quality of life in patients with old osteoporosis-related vertebral fracture.

Uebelhart et. al. believe that in addition to drugs, treatment of chronic back pain due to osteoporosis should also include physical agents, occupational therapy and stabilizing methods [2].

Zambito et al., in a randomized, double blind, clinical study, analyzed the effect of application of interferential currencies (IFC) and horizontal therapy $(\mathrm{HT})$ in patients with chronic lumbar pain due to previous multiple vertebral osteoporotic fractures in 115 female patients. The patients were divided in three groups, the first group received IFS, the second $\mathrm{HT}$, and the third one placebo HT. They received therapy 30 minutes a day, 5 days a week, for a period of 2 weeks, along with an exercise program. Patients were evaluated before treatment, in 2, 6 and 14 weeks by Visual Analog Scale (VAS) for pain, Backill questionnaire for disability and medium application of analgesics. The results after 2 weeks showed considerable and similar improvement of VAS and Backill score in all three groups. Both scores were considerably better in patients from the two groups receiving active therapy for 6 and 14 weeks. The use of analgesics was better in the HT group only. The results referred to the evidence that IFS and HT were considerably efficient in reducing pain caused by previous multiple vertebral fractures [23].

Rossini $\mathrm{M}$ et al. investigated the effect of capacitively coupled electrical stimulation (CCES) in treatment of female patients with pain caused by vertebral compressive osteoporotic fractures. CCES is a type of electrical therapy approved by the US FDA as a method for enhancing of bone healing and for spinal fusion. In the randomized clinical trial (RCT), 51 women in post-menopause with multiple fractures and chronic pain were involved. The study lasted for 3 months. The VAS for pain and the score of the Quality of life Questionnaire of the European Federation for Osteoporosis (QUALEFFO) was improved in two groups. Capacitively coupled electrical stimulation was less effective than the controlled treatment depending on the hours of treatment. In the group receiving CCES, majority of patients could discontinue application of NSAIL due to elimination or reduction of pain in comparison with the patient in the control group. The authors have concluded that CCSE has effects on the pain control in some patients and that it reduces application of NSAIL [24].

In a systematic review on effectiveness and safety of Vitamin D in relation to bone health (167 studies of which 112 were RCTs), the importance of ultraviolet B rays (artificial or with exposure to sunlight) was analyzed in 8 RCTs. Studies were heterogeneous in respect to accurate UV-B doze determination and examination of $25(\mathrm{OH}) \mathrm{D}$, but radiation showed positive effect on serum concentration of $25(\mathrm{OH}) \mathrm{D}$. However, the degree of sunlight exposure that would be sufficient to maintain serum concentration on $25(\mathrm{OH}) \mathrm{D}$ was not analyzed, and on the other hand, it minimizes the risk of melanoma or other skin tumor [25].

\section{Conclusion}

To complement pharmacological therapy and exercises applied in the complex treatment of patients with osteoporosis, specific physical agents should also take their place and contribute in reducing subjective symptoms, improving clinical findings and quality of life. However, further clinical studies are required to achieve a better record on their application. 


\section{References}

1. Bonner Jr.FJ, Chesnaut $\mathrm{CH}$, Lindsay R. Osteoporosis. In Physical Medicine and Rehabilitation. Principles and Practice. $4^{\text {th }}$ Edition, Ed. DeLisa JA, Lippincott Williams and Wilkins, 2005:699-719.

2. Uebelhart B, Rizzoli R. Osteoporose et douteur ou I'osteoporose fait-elle mal? Revue Medicale Suisse. 2005;22 (25):1662-5.

3. Silverman SL, Piziak VK, Chen P, Misurski DA, Wagman RB. Relationship of health related quality of life to prevalent and new or worsening back pain in postmenopausal women with osteoporosis. The Journal of Rheumatology. 2005;32(12) 2405-9.

4. Hadjipavlou AG, Lander PH. Osteoporosis of the spine and its management. In Spine care, Eds White A., Mosby, 1995:847869.

5. Zehnacker $\mathrm{CH}$, Bemis-Dougherty A. Effect of weighted exercises on bone mineral density in post menopausal women. A systematic review. J.Geriatr Phys Ther. 2007; 30(2):79-88.

6. Bonauti D, Shea B, lovine R. Et al. Exercise for preventing and treating osteoporosis in postmenopausal women. Evid Based Nurs. 2003;6(2):50-1

7. Asikainen TM, Kukkonen-Harjula K, Miilunpalo S. Exercise for health for early postmenopausal women: a systematic review of randomized controlled trials. Sports Med. 2004;34(11):75378.

8. Raskova M., Kirova I. Osteoporoza. In Fizikalna terapia, Arso: Sofia, 2002:234-235.

9. Preisinger E. Die Physikalische Therapie bei Osteoporose. Wien Med Wochenschr. 1994;144:612-8.

10. Nikolikj-Dimitrova E. Osnovi na fizikalna terapija. Second edition, Laserdzet:Skopje, 2011.

11. Liani-Galvao AP, Lazaretti-Castro M. Physical approach for prevention and treatment of osteoporosis. Arg Bras Endocrinol Metabol. 2010;54(2):171-8.

12. Kotel'nikov GP, Piatin VF, Bulgakova SV, Shirolapov IV. Whole body vibration (acceleration) training increases bone mineral density and serum levels of osteocalcin in elderly women. Adv Gerontol. 2010;23(2): 257-62

13. Uhlemann C., Lange U. Differenzialindikative physikalische therapiestrategien der odteoporose - empfehlungen fur den praxisalltag. Z.Rheumatol. 2006; 65(5):407-10.

14. Rubin CT, McLeod KJ, Lanyon LE. Prevention of osteoporosis by pulsed electromagnetic fields. J bone Joint Surg Am. 1989; 71(3):411-7.

15. Sert C, Mustafa D, Duz MZ, Aksen F, Kaya A. The preventive effect on bone loss of $50-\mathrm{Hz}, 1 \mathrm{mT}$ electromagnetic field in ovariectomized rats. J Bone Miner Metab. 2002;20(6):345-9.

16. Chang $\mathrm{K}$, Chang $\mathrm{WH}$. Pulsed electromagnetic fields prevent osteoporosis in an ovariectomized female rat model: a prostaglandin E2- associated process. Bioelectromagnetics. 2003; 24(3):189-98.

17. Li JK, Lin JC, Liu HC, Chang WH. Cytokine release from osteoblasts in response to different intensities of pulsed electromagnetic field stimulation. Electromagnetic Biology and medicine. 2007; 26(3):153-65.

18. Shen WW, Zhao JH. Pulsed electromagnetic fields stimulation affects BMD and local factor production of rats with disuse osteoporosis. Bioelectromagnetics. 2010;31(2): 113-9.

19. Stein E, Koehn J, Sutter W, Wendtland G, Wanschitz F Thurnler D. et al. Initial effects on differentiation of human osteoblast-like cells. Wien Klin Wochens-chr. 2008;120:112-7.
20. Stain A.,Beneyahu D, Maltz L, Oron U. Low-level laser irradiation promotes proliferation and differentiation of human osteoblasts in vitro. Photomed laser Surg. 2005;23:161-6.

21. Albertazzi $P$, Steel SA, Bottazzi M. Effect of intermittent compression therapy on bone mineral density in women with low bone mass. Bone. 2005;37(5):662-8.

22. Huang LQ, He HC, He CQ, Chen J, Yang L. Clinical update of pulsed electromagnetic fields on osteoporosis. Chinese Medical Journal. 2008;121(20):2095-9.

23. Zambito A, Bianchini D, Gatti D, Rossini M, Adami S, Viapiana $O$. Interferential and horizontal therapies in chronic low back pain due to multiple vertebral fractures: a randomized, double blind, clinical study. Osteoporosis Int. 2007;18(11): 1541-5.

24. Rossini M, Viapiana O, Gatti D, de Terlizzi F, Adami S. Capacitively coupled electric field for pain relief in patients with vertebral fractures and chronic pain. Clin Orthop Relat Res. 2010;468 (3): 735-40.

25. Cranney A, Horslay T, O'Donnell S, Weiler H, Puil L, Ooi D, Atkinson S. et al: Effectiveness and safety of vitamin $D$ in relation to bone health. Evidence Report/Technology assessment. 2007;158:1-235. 